\title{
Introduction To Rotor Eddy Current Loss Of Permanent Magnet Motor For Electric Vehicles
}

\author{
Shoujie Tang \\ Department of Electric Engineering \\ Shanghai Dianji University \\ Shanghai, China \\ shoujie2012@sina.com \\ Hairong Zou \\ Department of Electric Engineering \\ Shanghai Dianji University \\ Shanghai, China \\ zouhr@sdju.edu.cn
}

\author{
Wan Liu \\ Department of Electric Engineering \\ Shanghai Dianji University \\ Shanghai, China \\ 952060547@qq.com \\ Peng Wang \\ Department of Electric Engineering \\ Shanghai Dianji University \\ Shanghai, China \\ 876061170@gq.com
}

\begin{abstract}
In permanent magnet motor, the rotor eddy current loss can make the rotor temperature rise, affect the efficiency of motor performance and even lead to permanent magnet demagnetization. In order to improve the efficiency of the motor and protect the magnet which is core part of the motor, some ways must be adopted to optimize the motor to solve the problem of eddy current. According to the problem of eddy current loss of permanent magnet motor, the principle of eddy current loss was simply analysed in this paper. The eddy current loss can be influenced by the shape of magnet and rotor surface, structure of rotor, slit width of the stator, rotor shield (sheath), air-gap length etc. Some methods of optimizing the eddy current loss were introduced for the further study of the eddy current loss. It is also helpful for the efficiency research of the motor for electric vehicles.
\end{abstract}

Keywords- permanent magnet motor; demagnetization; eddy current loss; optimize; efficiency (key words)

\section{INTRODUCTION}

With the problem of population, lack of resources and environmental pollution in current days, people are faced with the challenge of the severe shortage of oil resources. Oil resource shortage is one of the major problems urgently needed to be solved in today's world. Vehicle emissions also become a great damage to the environment. The electric car is a kind of traffic tools with electric drive and gets rid of the traditional fossil energy demand. Therefore, development of the electric car is the effective way of solving the oil crisis and increasingly serious environmental pollution [1]. So it is inevitable to replace the internal combustion engine car by the new energy vehicles.

Permanent magnet synchronous motor has high power density, smaller volume and no additional rotor copper loss. Besides, it works more efficiently and needs less maintenance cost. In recent years, permanent magnet motor, especially for the built-in permanent magnet motor, has been a hot research topic in the field of electric vehicles and other industries which need high power density[2]. However, in order to prevent dust, water, acid alkali and other foreign matter from the inside of the motor, motors typically adopt fully closed structure and require high degree of protection. Because of the fully enclosed structure, it can improve the reliability, reduce the maintenance workload and reduce noise. But at the same time, the internal heat of motor also cannot effectively dissipate due to the fully closed structure. As a result, it causes the high temperature of the stator armature, permanent magnet, the bearing and shaft [3].

The temperature rise of the motor can be divided into two parts. One part, which is made of stator iron loss, copper loss and so on, is from the stator. It occupies a large part of the total loss of the motor. The other part comes from the rotor. It consists of the rotor eddy current loss, wind friction loss and so on. Generally, it is only a little part of the total loss. Nevertheless, the rotor is inside the motor and it cannot use the same special water or air cooling channels as the stator, so the heat generated by the rotor loss will accumulate inside the rotor, which causes high temperature rise of the rotor, affects the structure strength of rotor, reduces motor efficiency and even leads to irreversible permanent magnet demagnetization when seriously [4].Thus it is necessary to reduce the rotor loss in the research of permanent magnet motor. It is accordingly indispensable to optimize the design parameters of the permanent magnet motor or improve the cooling performance of the motor.

\section{PRINCIPLE ANALYSIS OF EDDY CURRENT LOSS}

Eddy current of permanent magnet in the motor is a result of time and space harmonic components caused by stator cogging, non-sinusoidal distribution of 
magnetomotive force of stator windings and magnet, nonsinusoidal phase current of stator etc. in the air gap magnetic field. The heat generated by the eddy current loss cannot effectively spread out, which overheats the rotor and causes permanent magnet demagnetization.

\section{A. Time harmonics}

Using an AC synchronous motor as an example, through the proper mathematical change and some simplified assumptions, the stator voltage equation in the rotor coordinate system can be expressed as [5]:

$$
\begin{aligned}
& v_{q}=r_{s i} i_{q}+L_{q p} i_{q}+L_{d} \omega_{e i d}+M_{q f} \omega_{e i f}+M_{q t} p i_{t}+M_{q} \omega_{e} i_{s} \\
& v_{d}=r_{s i d}+L_{d p} i_{d}-L_{q} \omega_{e i q}+M_{d f p i f}-M_{d t w e i t}+M_{d s p i s}
\end{aligned}
$$

Where, f expresses equivalent rotor excitation coil; $\mathrm{t}$ and $\mathrm{s}$ respectively expresses short circuit coil in $\mathrm{d}$ and $\mathrm{q}$ axis when making permanent magnet conductivity model.

When ideal motor is in steady-state operation, time integral of (1) and (2) is zero. So it is the time constant. It means that magnetic field produced by the stator winding is relatively stationary to the rotor and will not produce any eddy current in the permanent magnet. But in fact, that magnetic field changes with time, so $\mathrm{d}$, $\mathrm{q}$ axis current also changes with time. As a result, the eddy current loss will still be produced in permanent magnet.

Transfer a, b, c three phase current into d, $\mathrm{q}$ axis current:

$i_{a}=I \cos \theta, i_{b}=I \cos \left(\theta-120^{\circ}\right), i_{c}=I \cos \left(\theta+120^{\circ}\right)$

Where, $\theta=\theta_{0}+\omega^{\prime} t$, according to the (3), we can get the (4):

$$
\begin{aligned}
& i_{d}=I \cos (\alpha-\theta)=I \cos \left[(\alpha 0-\theta 0)+\left(\omega-\omega^{\prime}\right) t\right] \\
& i_{q}=I \sin (\alpha-\theta)=I \sin \left[(\alpha 0-\theta 0)+\left(\omega-\omega^{\prime}\right) t\right] \\
& i_{0}=0
\end{aligned}
$$

As a result, the harmonic component of the stator winding current can make ${ }^{i} d$ change with time. From (4), we can know that the frequency of $d, q$ axis current is $\omega-\omega^{\prime}$. So it will produce eddy current loss in the magnet.

\section{B. Space harmonics}

Ideally, the magnetic field of the rotor in air gap is distributed in sine. But in fact, because of the limitation of manufacturing process of permanent magnet, the harmonic content in permanent magnet rotor magnetic field is very large. Besides, stator windings also produce harmonic content in air gap, and they are all not relatively stationary to the rotor, so they all can produce eddy current loss on the rotor [6].

Make a Fourier transform for magnetic field of rotor:

$$
B_{r}(\theta)=\sum_{i=1}^{\infty} B_{2 i-1} \cos [(2 i-1) \theta]=B_{1} \cos \theta+B_{3} \cos 3 \theta+\mathrm{L}
$$

Where, $\theta$ is the electric angle between the axis of A phase and $\mathrm{d}$ axis. Similarly, stator windings also produce harmonic content, no more introductions here.

\section{OPTIMIZATION METHODS OF THE EDDY CURRENT LOSS}

For a long time, domestic and overseas scholars have done a lot of research on how to reduce the eddy current loss. Fundamentally, they all focus on the air gap flux density. According to the description above, we can know that the eddy current loss is caused by harmonic of air-gap flux density, so optimizing the air gap flux density can indirectly optimize the eddy current loss. In this paper, a brief summary of optimal method was made.

\section{A. Optimization of magnet of surface mounted motor}

For surface mounted permanent magnet motor, the rotor structure is usually as shown in Fig. 1 (a). Waveform produced by this kind of magnetic pole structure is rectangular, so the air gap flux density contains much higher harmonics. As shown in Fig. 1 (b), reference [7] made an optimization for this kind of magnetic pole structure, and put forward a new kind of structure using multiple pieces of permanent magnet instead of the conventional single piece. In this way, waveform of air gap flux density can be effectively improved.

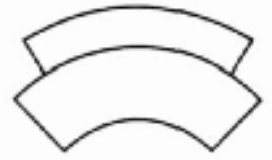

(a)

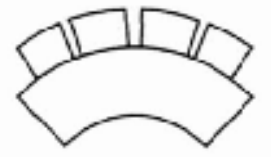

(b)
Figure 1. Rotor structure schematic diagram.

Magnet segmenting is one of the effective ways to solve the magnet eddy current loss. Reference [2] respectively studied eddy current loss of magnet segmented along the circumferential direction and axial direction, and got the relationship between the eddy current loss with and without segmenting. As shown in (6) and (7), (6) is along the circumferential direction and equation (7) is along the axial direction.

$$
\begin{gathered}
\frac{P_{\text {seg }}}{P_{\text {noseg }}}=\frac{\frac{\left(d\left(\frac{\psi}{N}\right)\right)^{2}}{2(R L+N R W)}}{\frac{(d \psi)^{2}}{2(R L+R W)}} \approx \frac{(W+L)}{\left(L N+W N^{2}\right)} \\
\frac{P_{\text {seg }}}{P_{\text {noseg }}}=\frac{\frac{\left(d\left(\frac{\psi}{N}\right)\right)^{2}}{2(R W+N R L)}}{\frac{(d \psi)^{2}}{2(R L+R W)}} \approx \frac{(W+L)}{\left(W N+L N^{2}\right)}
\end{gathered}
$$

Here, $N$ is the number of magnet segments, $\psi$ is the magnetic flux values passing through the magnet along the vertical direction. $W, L, R L, R W$ respectively expresses the width, axial length and the equivalent resistance value of the whole piece magnet steel. It can be seen from the equations above that the more the number of segments is, the smaller the eddy current loss is. 


\section{B. Optimization of rotor of interior permanent motor}

Surface mounted motor can change the shape of magnet to make the air gap flux density close to sine distribution. But, for the interior permanent magnet motor, magnet always uses general type of shape because of the built-in magnet. So, usually, rotor surface shape or the internal rotor structure is optimized to achieve the purpose of making air gap flux density distributed in sine. In [8], as shown in Fig.2, nine pairs of variables which control the rotor surface shape were determined by using b-spline curve.

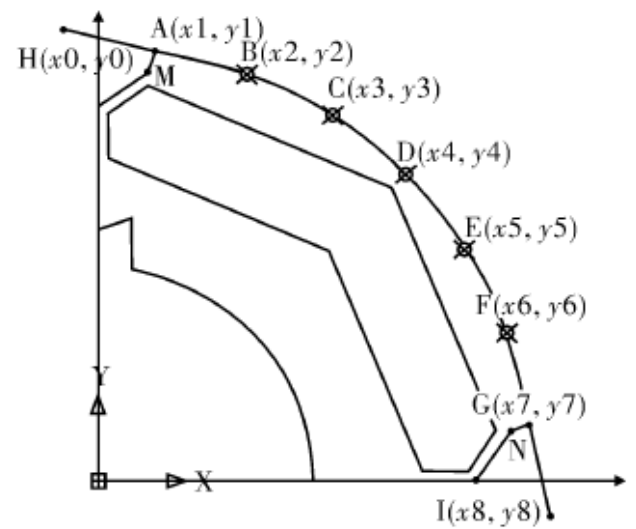

Figure 2. Design variables of rotor surface.

And then control variables were optimized to search the minimum value of the following (8). Where, $V$ expresses polygon vertices, $B k$ is the $\mathrm{k}$-th space harmonics of the air-gap flux density, $\delta$ is the length of the air gap, $\delta$ min is the minimum length allowed of air gap. After optimization, the harmonic content of air gap flux density is effectively reduced.

$$
\min f(V)=\min \sum_{k=2}^{n} B_{k}^{2}
$$

$\delta(V) \geq \delta \min$

In [9], the internal rotor structure of IPM was optimized with adaptive finite element method. Similarly, (9) was used as the objective function. Where, $\mathrm{k}$ is the order of space harmonics, $W$ is the harmonic losses.

$$
W=\sum(k B k)^{2}
$$

As shown in Fig.3, 15 design variables of rotor structure and magnet were closed to have an optimization.

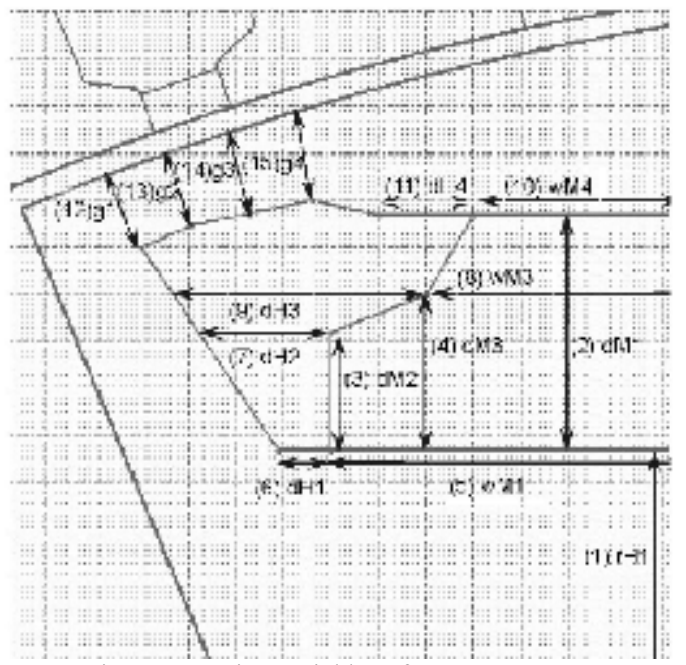

Figure 3. Design variables of rotor structure.

In [10], it was found that different positions of magnet at the rotor have influences on eddy current loss of permanent magnet. And a conclusion was got that eddy current loss was reduced the most when magnet was placed in the V-type position as shown in Fig.4.

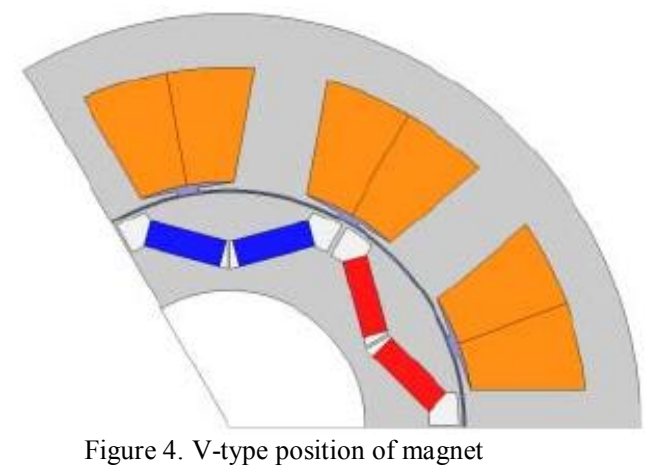

\section{Other optimizing ways}

In addition to the optimization methods said above, there are also many other optimization methods. Slit width of the stator, rotor shield (sheath), air-gap length etc. all have certain influence on eddy current loss. Reference [11] discussed the relationship between the eddy current loss and slit width of the stator and came to the conclusion that the decrease of the slit width can reduce permanent magnet eddy current loss. In addition, the number of stator slot also has a certain influence on eddy current loss.

In [12], the influence of sleeve circumferential grooves on the rotor eddy-current loss was analyzed. It was found that circumferential grooves could effectively reduce the rotor eddy current loss, improve the rotor cooling conditions and reduce the rotor temperature rise.

In [13], the relationship between the eddy current loss and air-gap length was discussed. It was described that the rotor eddy current loss decreases with the increase of air gap length, however, when the air gap length increases to a certain extent, the rotor eddy current loss will reduce slowly. At the same time, increasing the air gap length will accordingly reduce air gap flux density. In order to guarantee the air-gap flux density, the dosage of the permanent magnet must be increased, which increases the cost of the motor. So we have to weigh the pros and cons. 
Besides, and it also put forward that auxiliary slots on the stator tooth can effectively reduce the eddy current loss.

\section{CONCLUSION}

In this paper, the principle of eddy current loss was simply analyzed. It was caused by time harmonics and space harmonics in the air gap flux density. Some methods of optimizing the eddy current loss of the permanent magnet were introduced for the further study of the eddy current loss. There are optimization of magnet of surface mounted motor, optimization of rotor of IPM and other ways including optimizing the slit width of the stator, rotor shield (sheath), air-gap length etc.

\section{ACKNOWLEDGMENT}

The research work was supported by National Science Foundation of China under Grant No.51377104 and Production-study-research cooperation projects of Minhang District Shanghai city under Grant No. 2013MH109.

\section{REFERENCES}

[1] Chan, C. C., Bouscayrol, A., \& Chen, K. (2010). Electric, hybrid, and fuel-cell vehicles: Architectures and modeling. Vehicular Technology, IEEE Transactions on, 59(2), 589-598.

[2] ZHANG, L., \& GAO, C. X. (2013). Eddy-current loss model and index for magnets of permanent magnet synchronous motors. Electric Machines and Control, 7, 007.

[3] Siyuan He. Design of the cooling system for fully enclosed permanent magnet synchronous traction motor (In Chinese). High Power Converter Technology , 2012, (3): 48:52

[4] Jang, S. M., Cho, H. W., Lee, S. H., Yang, H. S., \& Jeong, Y. H. (2004). The influence of magnetization pattern on the rotor losses of permanent magnet high-speed machines. Magnetics, IEEE Transactions on, 40(4), 2062-2064.
[5] Juan Li. (2005). FEM analysis on eddy current losses in the permanent magnets and its influence on the machines' performance (In Chinese) [D].24-27(Doctoral dissertation, Tianjin: Tianjin university).

[6] Jingcan, L., \& Yong, L. (2011). Model of permanent magnet synchronous motor considering saturation and rotor flux harmonics. Proceedings of the CSEE, 31(3), 60-66.

[7] Luo, D., Cheng, B., Huang, S., \& Gao, J. (2009, November). Method for optimize the air gap flux density of permanent magnet synchronous motor. InElectrical Machines and Systems, 2009. ICEMS 2009. International Conference on (pp. 1-4). IEEE.

[8] Xiaohong Wu, Guangyou Li, \& Qinghe Zhu. (2014). Optimization of rotor shape of interior permanent synchronous motor (In Chinese). Micromotors, 47(4), 17-20.

[9] Yamazaki, K., Ohki, S., Nezu, A., \& Ikemi, T. (2007, May). Development of interior permanent magnet motors reduction of harmonic iron losses by optimizing rotor structures. In Electric Machines \& Drives Conference, 2007. IEMDC'07. IEEE International (Vol. 1, pp. 489-494). IEEE.

[10] Oh, S. Y., Cho, S. Y., Han, J. H., Lee, H. J., Ryu, G. H., \& Kang, D. (2014). Design of IPMSM Rotor Shape for Magnet EddyCurrent Loss Reduction.Magnetics, IEEE Transactions on, 50(2), 841-844.

[11] Chengning Zhang, Xiaopeng Wu, Yugang Dong, \& Fang Wang. (2013). Effect of slot opening on eddy current of permanent magnet in high power permanent magnetic synchronous motor driven by inverter (In Chinese). Transactions of Beijing Institute of Technology, 33(012), 1239-1242.

[12] Shen, J., Hao, H., \& Yuan, C. (2012, December). FEA study on circumferential grooves on rotor retaining sleeve of high-speed PM brushless motors. In Zhongguo Dianji Gongcheng Xuebao(Proceedings of the Chinese Society of Electrical Engineering) (Vol. 32, No. 36, pp. 53-60). Chinese Society for Electrical Engineering.

[13] Pengfei Gao, Jiancheng Fang, Bangcheng Han, \& Jinji Sun. (2013).Analysis of rotor eddy-current loss in high-speed permanent magnet motors (In Chinese) .Micromotors, 46(5), 5-11. 